Cultures \& Conflits

03 | automne 1991

Mafia, drogue et politique

\title{
Camorra et politique : démystification du rôle de la drogue
}

\author{
Maria-Luisa Cesoni
}

\section{(2) OpenEdition}

1 Journals

Édition électronique

URL : http://journals.openedition.org/conflits/107

DOI : $10.4000 /$ conflits. 107

ISSN : $1777-5345$

Éditeur :

CCLS - Centre d'études sur les conflits lilberté et sécurité, L'Harmattan

Édition imprimée

Date de publication : 17 octobre 1991

ISSN : 1157-996X

Référence électronique

Maria-Luisa Cesoni, «Camorra et politique : démystification du rôle de la drogue », Cultures \& Conflits [En ligne], 03 | automne 1991, mis en ligne le 24 février 2005, consulté le 30 mars 2021. URL : http:// journals.openedition.org/conflits/107 ; DOI : https://doi.org/10.4000/conflits.107

Ce document a été généré automatiquement le 30 mars 2021.

Creative Commons License 


\title{
Camorra et politique : démystification du rôle de la drogue
}

\author{
Maria-Luisa Cesoni
}

1 La mafia ne s'est pas développée par ses propres vertus. Elle a trouvé dans l'affaiblissement de la démocratie un facteur supplémentaire à son expansion. Pour ces raisons, la question mafieuse coïncide largement avec la question de la démocratie et peut devenir un facteur constitutif de notre système politique. (Commission parlementaire anti-mafia).

2 On parle couramment de la mafia italienne, comme s'il s'agissait d'un phénomène homogène. Cette image s'est tellement constituée en stéréotype qu'on appelle les différents phénomènes sociaux qui s'en rapprochent dans certains pays ou au niveau international, des mafias, comme si le modèle italien avait été tout simplement exporté.

Or, ce qu'on définit, en simplifiant, comme mafia, est, déjà en Italie, une réalité composée de plusieurs phénomènes(1). L'unification tardive de l'Italie a déterminé la persistance de zones très diversifiées, socialement, économiquement et culturellement. Par conséquent, les organisations de type mafieux ont eu une évolution et ont développé des caractéristiques hétérogènes, selon les régions. On peut donc les différencier non seulement à cause de leurs caractéristiques particulières, mais aussi du point de vue géographique et du contexte socio-économique.

4 A la dénomination imprécise de mafia correspondent ainsi des réalités différentes et il faut distinguer en fonction de leur implantation trois principaux types d'organisation : la mafia sicilienne, la `ndrangheta calabraise, la Camorra napolitaine.

5 Certes le banditisme sarde et les organisations criminelles des régions du nord représentent deux autres types d'organisation qui se rapprochent du type mafieux, cependant, le banditisme sarde ne peut être ramené simplement à un modèle mafieux, en raison de ses caractéristiques très particulières qui tiennent à son enracinement dans la précarité des ressources et les problèmes économiques de sa région, la Barbagia, plus aigus qu'ailleurs, et de ses forts liens avec la structure sociale sarde. Quant aux organisations $\mathrm{du}$ nord $\mathrm{du}$ pays, on connaît encore trop sommairement leurs caractéristiques et leurs liens avec les organisations de type mafieux du Mezzogiorno 
pour pouvoir les analyser. Il semble cependant qu'elles soient nées récemment (elles se seraient développées de manière importante à partir des années 70 et 80 ) et que leurs activités aient concerné, dès le départ, les secteurs économiques et financiers.

6 La mafia au sens strict naît donc au XIXe siècle en Sicile. Ses caractéristiques principales sont strictement liées au contexte socio-culturel de l'île : sens de l'honneur, de la famille, de la solidarité. Si, au début, elle avait aussi un rôle de médiation entre les gens du peuple et l'Etat (en offrant, surtout, protection et possibilités de travail), elle se constitue ensuite de plus en plus en centre de pouvoir.

De la Sicile, sa puissance se répandra très loin, notamment aux Etats-Unis.

8 La 'ndrangheta, localisée en Calabre, correspond à peu près au modèle de la mafia sicilienne, le tissu social étant semblable. Mais son développement sera plus lent, et conserve encore des résidus d'activités criminelles non modernes. Elle semble, ces dernières années, accélérer le processus de contrôle des activités économiques et d'infiltration dans la vie politique locale, ce qui a induit une escalade de la violence interne et externe.

9 La camorra, enracinée dans la réunion de Naples, est au départ un phénomène différent des deux autres. Tout d'abord parce que mafia et 'ndrangheta naissent strictement imbriquées dans le tissu social rural, tandis que la camorra se développe dans un contexte essentiellement urbain (Naples et son arrière-pays, ainsi que dans les provinces de Salerne et de Caserte). Ensuite, si les chefs mafieux représentent souvent de grandes familles locales, la plupart des camorristes les plus connus ne sont, au début de leur carrière, que de petits voleurs de rue. Enfin, si le recrutement des mafieux passe à travers les liens de la "famille" au sens large, comprenant aussi les liens de clan, les terrains privilégiés de recrutement des camorristes sont la rue et la prison.

Néanmoins, au-delà des différences originelles, la caractéristique commune à toutes ces organisations est une capacité élevée d'adaptation aux changements sociaux et économiques. En effet avec le temps, les activités illégales se sont déplacées du champ traditionnel des délits dits d'honneur, en passant par la prostitution, l'extorsion de fonds ou le jeu de hasard, vers le champ des délits économiques, ouvrant la voie aux investissements dans des activités économiques licites.

11 Malgré des ressemblances extérieures et des tendances à l'homogénéisation de ces phénomènes, la structure de leurs relations avec la société, la sphère économique et le pouvoir a donc été, jusqu'à présent, différente.

12 Nous étudierons plus spécifiquement la camorra, qui a retenu, bien après la mafia, l'attention des commissions d'enquête et du public, et qui paraît actuellement poser des problèmes institutionnels croissants, par son infiltration dans les administrations locales.

13 Nous essayerons, notamment, de montrer comment les "drogues illicites" n'ont été que l'un des facteurs multiples, qui fondent la force actuelle de la camorra, et dont le plus important réside dans ses relations avec la sphère politico-administrative.

La camorraLa camorra traditionnelle

14 L'histoire de la camorra traditionnelle se poursuit assez tard et jusqu'à une date récente, celle du milieu des années 1970. Ces années sont d'ailleurs identifiées par les spécialistes comme celles des "grands changements", et tout particulièrement celle du tremblement de terre (1980). 
D'après les archives de police, les premières traces officielles de la camorra datent de 1819-1820. Ce phénomène est cependant enraciné dans les changements économiques et sociaux qu'a connue Naples au cours du XVIIIème siècle(2). Les hypothèses formulées sur les raisons de sa naissance sont contrastées. Selon certains auteurs, la camorra est le produit du passage à l'acte illégal facilité par le relâchement de la discipline sociale provoqué par l'éloignement progressif de l'administration locale par rapport à la population. Selon d'autres auteurs, il s'agit plutôt d'un principe d'auto-discipline à l'égard de la violence provoquée par la misère(3). Il nous semble surtout pouvoir y discerner une manifestation déviée de l'insatisfaction sociale, bien moins dangereuse pour les autorités qu'un soulèvement populaire. Car, à Naples comme ailleurs, le pouvoir du peuple s était jusqu'alors manifesté par des explosions soudaines. Or, comme nous allons le voir, avec la camorra cette violence va être canalisée vers d'autres activités. Bien que plusieurs auteurs du XIXe siècle aient décrit les caractéristiques "organisationnelles" de la camorra, il n'y a pas de consensus généralisé sur son caractère d'organisation secrète.

Plutôt que d'insister sur ce caractère, il nous semble important d'en retenir deux autres. Le premier est que la camorra "représenta la seule forme de mobilité dans une structure sociale entièrement close"(4). Le second est que la camorra a été utilisée à plusieurs reprises par la police ou les autorités locales pour contrôler l'ordre citadin. En 1860, par exemple, le préfet Liborio Romano recruta dans la police les chef les plus réputés de la camorra, afin de rétablir l'ordre dans la ville, bouleversé par de très violentes émeutes.

La camorra était présente dans tous les quartiers de Naples, mais plus massivement dans les quartiers populaires. Son activité principale consistait dans le prélèvement d'un pourcentage sur toutes les activités, légales et illégales, auxquelles elle garantissait une protection. Elle ne cherchait pas en cela à soustraire la richesse des plus nantis, mais s'adressait plutôt à sa propre base sociale, pour laquelle elle jouait un rôle de garant de l'ordre dans les transactions sociales et économiques. I. Sales parle de "levée d'un impôt".

18 L'organisation se posait ainsi en représentante des couches populaires, pour qui elle était bien plus légitime qu'un État imposant lui aussi de lourds prélèvements mais sans rien donner en contrepartie. En effet, la camorra était le seul moyen de relation et de représentation politique du peuple, d'autant plus que ce dernier, jusqu'en 1912, était coupé de toute possibilité d'expression par le refus du droit de vote aux analphabètes. La camorra trouve donc son origine dans le peuple et y reste liée, bien qu'avec des variations d'intensité, au moins pendant le premier siècle de son histoire.

19 L'exercice de la violence est, aussi à l'époque, bien maîtrisé. La vaste légitimité populaire, unie à des signes extérieurs rendent immédiatement perceptible le rôle rempli par la camorra. Dès lors, le camorriste a très rarement à recourir à la violence : sa seule présence et sa parole suffisant à faire respecter l'ordre. La violence était ainsi un instrument de pouvoir à la fois symbolique et réel et, au moins à cette époque, plus symbolique que réel.

20 En ce qui concerne les activités illégales des membres de l'organisation, elles se limitaient aux délits normalement perpétrés sans recours particulier à la violence. Lés actes de violence, quand ils étaient nécessaires, étaient donc très ritualisés. 
21 Une dernière caractéristique de la camorra traditionnelle est son caractère déjà urbain(5). Le développement original de la camorra, différent pendant plus d'un siècle de celui de la mafia, est donc déterminé dès le XIXe siècle par le contexte urbain dégradé, l'urbanisation massive, les attentes déçues par l'incapacité de la ville à subvenir aux besoins de sa population et l'industrialisation tardive, ralentie et marginale, ne permettant pas à la population de se constituer en classe ouvrière et de s'organiser par des représentations syndicales ou politiques.

Les premiers changements

Dès le départ, la camorra se forme et fonde donc son activité sur le marché très développé - licite et illicite - représenté par la ville et le port de Naples. Les premiers changements dans sa structure surviennent avec l'unification de l'Italie. Les camorristes sortent renforcés de leur expérience dans la police et commencent à organiser une vaste activité de contrebande et d'appropriation des taxes douanières en menaçant les fonctionnaires. Seulement, leur pillage des caisses publiques est tel que la réponse des autorités consiste cette fois en une répression massive.

Simultanément, les camorristes commencent à franchir les barrières de classe. Ils débutent une carrière dans différents métiers, essentiellement commerciaux, et voient par conséquent leurs conditions de vie s'améliorer, ce qui les rapproche des classes bourgeoises. Dès lors le lien avec la population s'affaiblit.

24 A partir de 1875, avec l'élargissement du suffrage électoral, la camorra commence à s'intéresser aux enjeux politiques; cependant, son rôle est encore celui de prestations de service et d'offre de votes en échange de divers privilèges et facilités.

$\mathrm{Au}$ début du XXe siècle, la situation se modifie à nouveau. La loi spéciale pour l'industrialisation de 1904, l'émigration massive vers les Amériques et l'assainissement de certaines parties du centre ville déterminent une amélioration des conditions socioéconomiques urbaines et des possibilités offertes à la population. La légitimité de la camorra s'amoindrit alors auprès des couches populaires, qui forment progressivement une classe ouvrière, et en même temps elle échoue partiellement dans sa tentative d'intégration aux couches supérieures, provoquant une période de marginalisation.

Cette marginalisation de l'organisation camorriste se transforme, avec le fascisme, en une quasi-disparition - ou invisibilité qui dure jusqu'aux années 1950. En effet, dans la situation d'affaiblissement dans laquelle la camorra se trouve, la complète récupération du monopole de la violence - tant au niveau réel que symbolique - par le gouvernement fasciste ne peut que la priver de son instrument de pouvoir.

L'évolution récente

Dans les années suivant la deuxième guerre mondiale, les phénomènes de pouvoir illégal ou de criminalité organisée ne correspondent pas encore à la camorra actuelle.

Les bombardements, qui avaient détruit toute l'infrastructure moderne ainsi que les premières usines, réduisirent la vie de la population à un état précaire de survie, dans un environnement urbain dégradé.

29 Rien d'étonnant donc à ce que se développe, à la libération, un marché noir des biens et des aliments amenés par les troupes américaines, notamment essence, tabac, chocolat, ainsi qu'une reprise massive de la contrebande.

30 La contrebande de tabac, qui continuera même après la reprise de la vie économique locale, devient bientôt le moyen principal de survie, voire le seul, pour une partie importante de la population, et la principale activité économique de la ville. 
Elle intéresse bientôt la criminalité internationale : la position centrale et l'importance du port de Naples en font l'une des plus importantes places d'importation illégale et de redistribution vers d'autres régions. Ainsi, à partir de 1948, "la contrebande commence à s'organiser sur des bases industrielles et à se référer à une organisation capillaire"(6). A l'intérieur de cette organisation, toutefois, la criminalité locale, en cours de réorganisation, ne joue plus qu'un rôle subordonné pendant presque deux décennies. $\mathrm{Au}$ cours des années cinquante, la camorra s'intéresse à d'autres activités. Son rôle est principalement de médiation entre production agricole, conserveries, marchés nationaux et internationaux, ainsi que de régulation des relations commerciales dans la région de Naples. Elle contrôle bientôt, outre les marchés de gros, celui du bétail, de la viande et du lait, s'assurant la gestion du monopole des fournitures aux administrations locales. Elle développe aussi une activité commerciale directe. Il s'agit, cependant, plus d'un ensemble de groupes indépendants que d'une organisation centralisée et hiérarchique.

Selon Pino Arlacchi, c'est à cette époque que la camorra entretient, pour la première fois, des relations avec la mafia, qui, agissant en tant que fournisseur des marchés agricoles de la région napolitaine, utilise les créneaux commerciaux des camorristes(7). Le développement des échanges mondiaux, la hausse importante de la demande de tabac et, en 1960, l'élimination de l'avantage de zone franche pour le port de Tanger, qui valorise encore plus la rareté des contrôles dans le port de Naples, font accomplir un bond en avant à la contrebande de tabac. Ce serait l'essor et l'internationalisation de cette activité, ainsi que la présence en Campanie d'importants chefs mafieux, qui auraient permis à la camorra de passer à un stade organisationnel supérieur et d'accroître considérablement son importance(8).

Cependant, puisqu'elle ne possède pas les capitaux nécessaires pour intervenir directement dans un marché de cette importance dont les profits, selon Arlacchi, correspondront, en début des années 70, à environ 80 milliards de lires de l'époque(9) la camorra doit, pour la première fois, s'allier à la mafia, qui sera dans cette activité en position hégémonique.

En effet, la mafia commence de son côté à ressentir la nécessité de réinvestir les capitaux accumulés par sa participation à la spéculation sauvage, qui a eu lieu à Palerme dans le secteur du bâtiment. La camorra, en revanche, d'une part n'a pas eu l'occasion de participer à des activités aussi rentables et, d'autre part, ne peut pas vraiment accumuler, ayant besoin de dépenser pour se garantir une légitimité sociale.

Mais la mafia elle-même se heurte aux clans marseillais et corses qui sont favorisés par une disponibilité financière plus élevée et qui recourent souvent à des investisseurs légaux. Dès lors se produit une situation d'oligopole, qui se conclura au début des années soixante-dix, lorsque la guerre entre mafieux et organisations criminelles françaises se terminera à l'avantage des premiers. Durant toute cette période, la camorra garde son rôle d'organisation locale du trafic et de fourniture de main d'oeuvre, mais elle reste subordonnée à la mafia(10).

L'intégration progressive du marché de la morphine et de l'héroïne à celui du tabac, en utilisant le même réseau, et le déplacement du centre du trafic de stupéfiants de Palerme à Naples, voient encore les familles siciliennes prévaloir sur les familles campaniennes. Naples devient ainsi le principal centre de redistribution de l'héroïne provenant de l'Est asiatique vers les USA et le Canada, et de la cocaïne péruvienne vers les marchés italiens et européens. 
Les facteurs de transformation : le trafic de stupéfiants et le tremblement de terre la plupart des importations et de la vente en gros de la morphine et de l'héroïne transitant par la Campanie. Ce sont elles qui gèrent aussi l'activité de contrebande et le trafic d'armes, mais cela concerne surtout la Nouvelle Famille. En revanche la Nouvelle 
camorra Organisée de Cutolo, se spécialise plutôt dans le trafic de cocaïne, qui n'est pas encore investi par la mafia dans les années 1975-1980.

Ainsi, au début des années quatre-vingts, la camorra possède enfin assez de capitaux et de légitimité pour pouvoir traiter avec la mafia sur un pied d'égalité. Un véritable accord aurait alors été conclu entre les deux organisations, attribuant le contrôle du marché de l'héroöne à la mafia et celui de la cocaïne à la camorra, tout en établissant des liens logistiques étroits.

Si le trafic de stupéfiants représente donc un moyen d'accumulation rapide pour l'ensemble des distributeurs intermédiaires, il n'est pas sans poser problème. En effet, avec l'introduction des drogues illicites sur le marché napolitain, l'attitude des couches populaires change, créant ainsi une zone d'illégalité qui n'est plus entièrement acceptée.

Ainsi, selon Isaia Sales, le trafic aurait provoqué une rupture partielle du consensus de la base sociale camorriste, qui aurait recommencé à développer les marchés parallèles de biens non dangereux, en voyant que l'usage d'héroïne se répandait parmi les jeunes, notamment dans les couches défavorisées(13). On assiste ainsi au retour d'une partie de la population à des activités illégales ou informelles - plus traditionnelles et légitimées. Il est en outre possible que le choix de la Nouvelle camorra Organisée, plus liée à la base populaire, de se spécialiser dans le trafic de cocaïne, ait représenté aussi une manière de conserver cette légitimité, cette substance étant consommée dans les milieux bourgeois plutôt que dans les milieux défavorisés et jouissant ainsi d'une moins mauvaise réputation.

Le contrepoids de cette perte de légitimité consiste cependant en une mobilité sociale accrue, car les capitaux issus du trafic de stupéfiants commencent à être investis dans d'autres secteurs, entraînant ainsi une diversification des activités de la camorra, sur les marchés informel et officiel.

Les profits du marché des stupéfiants sont en effet considérables. De plus l'activité de la camorra ne s'exerce pas uniquement sur ce marché. Par son implication dans les autres activités économiques illégales et ses investissements dans les secteurs informel et officiel, l'organisation offre de plus en plus d'emplois et opère des redistributions de revenus.

51 Elle développe aussi, selon la tradition, des activités à la fois redistributives et de solidarité : fortes dépenses dans les commerces des quartiers défavorisés, aides aux familles en difficulté, financement d'activités sportives.

52 Elle $\mathrm{n}$ offre pas seulement, à ses affiliés, un enrichissement facile, mais aussi des possibilités de mobilité sociale : et même les seules possibilités pour des individus qui, n'ayant aucune chance au départ, "de chômeurs, de déshérités, vont devenir en quelques mois commerçants, propriétaires d'auberges, de cafés, ou de restaurants"(14).

53 Enfin la faiblesse de la vie associative citadine fait de la camorra le seul agent qui garantisse la satisfaction des besoins essentiels pour une partie importante de la population. La Commission parlementaire d'enquête souligne le danger que le pouvoir de la camorra s'élargisse jusqu'à comprendre la tutelle des droits civils des citoyens, à cause du "marasme actuel de la justice civile"(15)

54 Il nous semble donc qu'il reste encore des raisons suffisantes, notamment économiques, pour déterminer une légitimation sociale diffuse de l'organisation camorriste. Ainsi dans l'aire napolitaine, 300000 personnes vivraient de la camorra(16). Au-delà du trafic 
de stupéfiants, elle semble donc destinée à perdurer, tant qu'il n'y aura pas de changement substantiel dans les conditions de vie d'une partie considérable de la population.

Structuration des organisations camorristes, prise d'indépendance à l'égard de la mafia et du pouvoir politique, mobilité sociale accrue, division du travail entre les deux différents types d'organisation (Nouvelle camorra Organisée et Nouvelle Famille) et diversification des secteurs d'investissement, telles sont les modifications majeures induites sur la camorra par le développement des marchés de stupéfiants.

Le tremblement de terre

Le tremblement de terre de 1980 est le deuxième facteur qui a déterminé d'importantes modifications dans la structure des activités camorristes et dans les équilibres existant entre la camorra se réduisant bientôt aux familles qui se sont regroupées dans la Nouvelle Famille - et le système politico-administratif local. Dans une économie comme celle du Mezzogiorno, déjà gérée selon une logique d'intervention publique, le cataclysme apporte brusquement une quantité considérable de nouvelles ressources financières publiques. Les allocations pour la reconstruction se multiplient entre 1981 et 1990 puisque les allocations pour le seul "programme extraordinaire de constructions résidentielles", initialement prévues pour un montant de 1500 milliards de lires, parviendront à 13500 milliards en 1988(17).

Comme le constatera une commission parlementaire d'enquête ad hoc, une partie importante de ces ressources financera d'énormes ouvrages inutiles, alors que des milliers de personnes se logeaient toujours dans des conditions précaires. En effet, très rapidement, la camorra est prête à profiter de ce flux d'argent. Par la constitution ou l'achat d'entreprises, elle est présente dès l'envoi des premiers secours: elle monopolise rapidement les secteurs essentiels pour la reconstruction, comme la fourniture de béton et de matériaux inertes(18).

La camorra intervient massivement dans les marchés publics, tout en n'y accédant qu'indirectement par l'imposition aux entreprises ayant gagné le marché d'achats ou de contrats de sous-traitance avec les entreprises camorristes. L'organisation réussit ainsi à drainer une partie apparemment importante des allocations publiques. Cela lui permet, selon certains magistrats, d'abandonner le contrôle de l'importation des drogues illicites aux organisations des pays producteurs, et celui de la distribution à des bandes locales indépendantes pour se concentrer sur la source de ses principaux profits : l'accaparement des marchés publics(19).

La camorra se transforme donc selon la définition d'Amato Lamberti en "holding économico-financier" et en "sujet politique" à partir de l'accumulation considérable produite, d'abord, par le contrôle d'une partie du marché des stupéfiants et, ensuite, d'une grande partie des marchés publics. Parallèlement à la relance d'activités illégales comme la contrebande, le jeu clandestin, la falsification des produits de marque, ses activités économiques légales se développent de plus en plus et concernent des secteurs de plus en plus diversifiés (construction, tourisme, commerce, services, conserverie et autres). Les camorristes peuvent ainsi passer des activités illégales aux activités légales, selon une mobilité accrue et avec une plus grande légitimité sociale parmi les milieux nouvellement intégrés.

60 Les deux modifications majeures induites par les flux financiers ayant suivi le tremblement de terre semblent donc être d'une part, un désinvestissement progressif du marché des stupéfiants et, d'autre part, un investissement accru des relations avec 
les milieux politico-administratifs, allant d'une coïncidence d'intérêt vers une coïncidence de personnes.

Les relations internationales des organisations de type mafieux

61 En 1989, le service antidrogue du ministère de l'Intérieur fait état d'une connexion entre criminalité organisée italienne et étrangère pour le trafic de stupéfiants, et d'une présence croissante en Europe des organisations criminelles extra-communautaires. Il constate aussi une augmentation considérable de la production de toutes les "plantes à drogue"(20).

A la fin de 1990, des sources judiciaires affirment que les équilibres seraient en train de se modifier. En ce qui concerne la mafia, jusqu'au début des années 1980, maîtresse absolue du marché italien et international de l'héroïne blanche et détentrice du monopole de la transformation de la morphine-base en héroïne, elle aurait perdu ce contrôle au cours de ces dernières années. Son pouvoir dans ce domaine serait récupéré par les triades chinoises et les yakuza japonais. De même, les cartels colombiens qui contrôlent la production de cocaïne seraient en train de supplanter les organisations italiennes, en assurant directement le transport et la livraison dans le pays : elles pourvoiraient, "presqu'en régime de monopole, aux opérations de transformation de la substance et de sa commercialisation en Amérique du nord et en Europe occidentale"(21).

La part de la mafia et de la camorra s'affaiblirait donc au bénéfice des organisations étrangères. Un accord pour l'utilisation de la Méditerranée comme plaque tournante du trafic des stupéfiants aurait été conclu entre mafia et trafiquants colombiens(22). Cette rationalisation progressive du marché correspond d'ailleurs à son internationalisation croissante, qui s'accompagne de l'émergence et du renforcement d'autres acteurs que les organisations de type mafieux italiennes. Cette émergence correspond aussi à la facilité particulière d'approvisionnement due à la surproduction, qui crée une surabondance de produits par rapport à la demande et rend plus difficile le maintien d'un monopole.

64 Mais la modification des équilibres serait due au fait que les organisations de type mafieux italiennes privilégieraient de plus en plus autres champs d'activité, dont l'accaparement des marchés publics. S'il n'existe pas d'informations précises sur les relations que la camorra entretient avec les organisations de type mafieux étrangères dans d'autres domaines que les stupéfiants, des indices font penser qu'elles existent, tout au moins au niveau logistique. Le développement et les imbrications des différents marchés illégaux internationaux commencent en effet à ressortir des enquêtes judiciaires, notamment en ce qui concerne des réseaux de vente armes et de drogues illicites, dans lesquels les organisations de type mafieux italiennes seraient intervenues comme médiateur. Par ailleurs, on a constaté l'installation à l'étranger de plusieurs membres reconnus de la camorra: en France, mais aussi en Amérique latine et en Afrique. Or, on sait qu'une partie des profits s activités de la camorra sont actuellement investis à l'étranger" ; sur les marchés financiers internationaux. I1 faut donc supposer i'une partie de ces implantations à l'étranger est due à la volonté contrôler certains investissements. Selon la Commission parlementaire d'enquête, certaines familles camorristes liées à la mafia, comme celle de Michele Zaza, auraient des intérêts dans plusieurs pays d'Europe, tels que la Suisse, la Grèce, la France, l'Angleterre mais aussi aux USA, au Moyen-Orient et en Amérique latine(23). Les collaborations directes entre la camorra et les organisations de type mafieux latino-américaines commencent à être 
confirmées par les enquêtes judiciaires. Aussi, des Italiens participeraient aux opérations de blanchiment des profits générés par les étapes africaines du trafic de stupéfiants, via les casinos: par exemple, une présence croissante d'éléments camorristes aurait été constatée en Ligurie, autour de Sanremo, où se trouve l'un des plus grands et des plus suspectés des casinos italiens.

Enfin, la gestion d'activités illégales à l'étranger, comme la contrebande de cigarettes à Marseille, dénoncée dans le procès contre Michele Zaza, en France lors de l'été 1991, peut difficilement se faire sans l'accord, voire la collaboration, des groupes criminels locaux.

La camorra et les institutions politico-administratives

Les deux dernières décennies sanctionnent ainsi le processus de "mafizzazione" de la camorra. En effet, dans les guerres qui se sont produites à Naples au début des années quatre-vingts entre la Nouvelle camorra Organisée de Cutolo et la Nouvelle Famille, liée à la mafia, c'est effectivement cette dernière qui gagne. Les maxi-procès qui se sont déroulés à Naples dans la même période, ont par ailleurs eu un rôle déterminant dans l'anéantissement de la Nouvelle camorra Organisée.

"La camorra est en train de devenir mafia", affirmait en 1983 Giovanni Palombarini, à l'époque secrétaire de Magistratura Democratica, le courant de gauche de la magistrature italienne. "En Campanie (ainsi qu à Reggio et en Sicile), un certain type de criminalité organisée, existant depuis longtemps, mais contenue dans certaines limites, et agissant au bas niveau des articulations économiques avec une fonction de subsistance pour ceux qui la pratiquaient, devient protagoniste et se propose de manière immédiate d'agir en tant qu'acteur économique, entrepreneur investissant des capitaux élevés, produisant de l'emploi. Par conséquent, touchant à la sphère politique, la camorra est poussée à se poser comme candidat à la gestion d'une partie du pouvoir politique lui-même"(24). La relation entre la camorra et les autorités politicoadministratives a traditionnellement été une relation d'échange de services, où la première avait une position subordonnée. En effet, comme nous l'avons vu, l'échange consistait, au départ, en une offre de votes et un contrôle de la violence urbaine de la part de la camorra, contre la tolérance de l'illégalité de la part des autorités locales. Ces dernières avaient alors intérêt, en considération de la précarité urbaine jamais vraiment résolue par les politiques publiques, à se servir de manière stable de la camorra comme garant du maintien d'un certain ordre, quitte à réprimer durement les camorristes quand celui-ci est mis en danger. A la différence de la mafia, qui a été utilisée comme instrument direct de contrôle social dans des occasions ponctuelles, la camorra a joué ce rôle de manière durable, dans une relation instrumentalisée aux activités de police. Seulement avec les changements intervenus dans les années 1970 et 1980 , la relation se modifie.

La tolérance de l'illégalité persiste, et elle est renforcée par deux événements : d'une part, par la perte du monopole de la violence autorités étatiques, due au développement simultané de différentes formes de violence, criminelle et politique; d'autre part, par tremblement de terre qui, s'inscrivant dans un environnement socioéconomique jamais assaini, a dégradé énormément le cadre de vie de la population urbaine. Le principal facteur de régulation des conflits urbains reste donc ou redevient à partir de ces années la camorra.

69 Le flux de ressources destinées à la reconstruction modifie en effet de manière substantielle les équilibres existant entre la camorra et le système politique local. La 
gestion politique et clientélaire des ressources et les intérêts camorristes dans la gestion de ces dernières coïncident si bien, qu'ils déterminent "une soudure entre le système illégal privé (camorra) et le système illégal public"(25). La camorra passe ainsi d'un rôle subordonné à un rôle paritaire, sinon prédominant, grâce notamment au monopole de la "ressource violence", qu'elle détient au détriment du système politique, et qui la rend gagnante lorsque la relation se situe dans le partage du domaine de l'illégalité (de la gestion publique). Les renforcements des conduites illicites induits dans les administrations locales par les subventions publiques d'après 1980 sont en effet déterminants.

70 Mais selon certains, la classe politique, à l'occasion des maxi-procès, aurait lutté pour regagner l'hégémonie sur le système de fonctionnement illégal des administrations locales. Cette répression aurait été provoquée, comme autrefois dans l'histoire, par le dépassement de la part de la camorra du seuil de tolérance, provoquant une forte alerte de l'opinion publique et menaçant la légitimité et les intérêts économiques du pouvoir politique(26). Ces procès, qui ont abouti à la destruction de la seule Nouvelle camorra Organisée, représentant un potentiel de déstabilisation par le bas, et non à celle de la Nouvelle Famille, plus lié à la sphère administrative et politique, semblent cependant avoir servi involontairement le processus de rééquilibrage des pouvoirs et des alliances. Rééquilibrage qui, d'ailleurs, paraît être le prélude à l'implication directe des camorristes dans le champ du politique.

71 Au-delà des effets indirects, il faut de toute manière constater, avec plusieurs magistrats et spécialistes du secteur, que la focalisation sur la seule intervention judiciaire, réduisant des problèmes complexes comme celui de la camorra à des problèmes d'ordre public et les reléguant ainsi dans les domaines de la police et de la magistrature, correspond à l'absence d'une volonté institutionnelle d'affronter les causes du phénomène par des interventions politiques, économiques et sociales.

Les administrations locales

Plutôt que d'en rester à cette simplification judiciaire, il faut en effet insister sur la complexification progressive des mécanismes, du contenu et du niveau de l'échange politique. L'institution de la Caisse pour le Mezzogiorno, en 1950, avait déjà produit un renforcement des institutions publiques et des administrations locales, "dont le caractère opérationnel dans le Mezzogiorno aurait été insignifiant sans la Caisse"(27).

Dans les années soixante-dix, avec le développement du système de welfare state et la décentralisation administrative, les administrations locales prennent une importance croissante dans les mécanismes de répartition des dépenses publiques, pouvoir qu'elles ne perdront pas dans la période suivante, malgré la crise du système providentiel et la réduction des dépenses, puisque même si leurs budgets sont réduits, la relative rareté des ressources renforce leur pouvoir. Ainsi, au cours des années soixante-dix et quatrevingts, la primauté économique, ou l'hyperfonction économique, des administrations locales méridionales s'est accentuée, parallèlement à la chute verticale d'autres possibilités économiques et productives... et c'est "autour des administrations locales et de leur fonction administrative croissante que s'est développé un type particulier de marché, le marché politique, qui a ordonné toute l'activité économique au politique, en sélectionnant politiquement le système des entreprises et en empêchant la formation de processus réels d'autonomie dans la société civile. Le pouvoir politique est ainsi devenu le régulateur absolu de la vie sociale et économique(28). 
74 Le cadre est donc celui d'une politique d'intervention étatique qui, au lieu de développer les potentialités du Sud, se fait de plus en plus par des voies d'assistance. Au cours des années 1970 et 1980 - alors même que la camorra développe sa logistique et multiplie ses activités - l'État abandonne progressivement toute velléité de politique cohérente pour le Mezzogiorno, et réduit ses interventions à une réponse aux situations exceptionnelles.

75 Ce processus accroît l'état de dépendance du Mezzogiorno vis-à-vis du reste du pays, sans parvenir à satisfaire les besoins essentiels de la population.

76 A partir de 1980, la nouvelle et considérable disponibilité de ressources, due aux subventions pour la reconstruction, aura un effet de renforcement encore plus fort. Depuis toujours créatrices d'emploi et distributrices de subventions, les collectivités locales responsables de la gestion d'importantes ressources financières assument de plus en plus un rôle d'agents économiques véritables surtout par rapport au développement réduit des secteurs productifs. Elles se substituent ainsi, de manière progressive, à la Caisse pour le Mezzogiorno et aux Participations d'État, principaux moteurs de l'industrialisation de base de la région. Parallèlement la décentralisation de compétences nombreuses et disparates produit une classe de fonctionnaires souvent non qualifiés, mais dotés d'un pouvoir accru. Une enquête, menée en 1984 par le Parti Communiste dans trente administrations municipales campaniennes, a révélé l'existence d'un système de détournement, dans le but de piloter les subventions et les adjudications des marchés publics. A cette fin, on utilisait l'organe chargé de la gestion extraordinaire et des décisions d'urgence en tant qu'instance de décision ordinaire. "Il s'agit là de milliers et de milliers de décisions concernant adjudications, fournitures, travaux publics, paiements, subvention qui furent soustraites à la discussion et à l'approbation des conseils municipaux mais furent rendues immédiatement exécutables. (...) C'est à travers ces décisions, que passa la plus grande partie de la gestion administrative"(29). En outre, de cette manière, on empêcha tout contrôle de la part des élus des partis d'opposition.

77 Ce dysfonctionnement, ou mieux fonctionnement illégal, permet alors que les activités publiques soient contrôlées par l'extérieur, ce qui correspond forcément à un déplacement du pouvoir en dehors des institutions. Politologues et magistrats ont bien analysé ce processus typique de l'Italie contemporaine, où les principaux mécanismes de régulation socio-économique se situent en dehors des institutions. On assiste ainsi à la formation de lobbies de pouvoir, dont certains sont clandestins et illégaux. Cette tendance "ne concerne pas des aires marginales ou sections 'déviantes' de l'État ou de l'administration publique mais, comme les listes de Gelli (loge P2) le démontrent de manière exemplaire, cela intéresse en profondeur et en étendue les modes mêmes d'organisation et de prises des décisions"(30).

Camorra et politique

78 Cette situation de "morcellement des pouvoirs au niveau national" ne peut que représenter un facteur d'incitation à la "formation de sociétés criminelles tendant à sortir des conditions de marginalité et de dépendance pour entrer dans le jeu politique (...), à gérer une ou plusieurs des nombreuses miettes de pouvoir et de clientèle jetées sur le marché par une gestion inconsciente des Institutions publiques (Cosa pubblica) (31)".

79 En effet, après la parenthèse des premières années soixante-dix, pendant lesquelles la notion d'intérêt collectif était prônée et promue par l'existence d'une opposition de 
gauche, il semble que ces valeurs se soient retranchées dans les instances sociétales et que le but des partis italiens soit redevenu la gestion du gouvernement en tant que lieu privilégié d'appropriation du pouvoir et des ressources. Les partis politiques tendent ainsi à se transformer eux-mêmes en groupes d'intérêts comme les autres, répondant souvent aux demandes des groupes de pression les plus forts(32) et pensant la politique comme l'occasion de conclure des affaires, "à cette seule différence qu'ils sont spécialisés dans la gestion de l'appareil institutionnel"(33).

Le lien entre partis politiques et administration publique est d'ailleurs direct. Il suffit de parcourir les polémiques sur les mécanismes de nomination aux fonctions dirigeantes dans l'administration publique relatées par la presse italienne récente pour s'en rendre compte: des instituts de crédit publics aux administrations des services locaux de santé "le monde politique italien est si conditionné par la loi du partage (lottizzazione) qu il a fini par la considérer comme la seule méthode possible pour les nominations dans le secteur public"(34).

81 Naples, où le clientélisme a hypothéqué le développement des partis politiques, représente un des terrains les plus favorables pour le déroulement du processus que nous venons de décrire. Avec les flux financiers et les modifications de l'appareil institutionnel local des années quatre-vingts, la culture et les intérêts de la camorra convergent vers ceux du pouvoir politique. Dans la structure économique particulière de Naples, ces deux "lobbies" représentent les principaux facteurs de mobilité sociale. La rareté des ressources financières, concentrées dans le secteur public, en fait le seul moyen d'enrichissement en dehors des activités criminelles. Le rapport qui s'instaure entre camorristes et hommes politiques est donc une alternance de conflits et d'accords pour l'hégémonie dans la gestion des ressources.

L'activité de la camorra investit en effet tout le domaine de l'"économie administrative". D'abord elle concerne, sinon le monopole, du moins le contrôle d'une partie consistante des fournitures aux institutions publiques. Les scandales relatifs aux sur-profits obtenus dans le secteur alimentaire par la vente de denrées détériorées aux prisons, aux hôpitaux et aux maisons de retraite dont certains, retentissants, remontent à 1été 1989, n'en constituent qu'un exemple.

S'il y a eu une participation traditionnelle de la camorra à l'activité de redistribution des subventions pour le Mezzogiorno octroyées à partir des années cinquante, par ses échanges de services avec les décideurs, avec les aides pour le tremblement de terre, elle intervient cette fois massivement, pour la première fois, dans la gestion des ressources destinées à 1'assistance publique. Cela lui permet d'orienter les subsides indemnités de maladie, pensions d'invalidité, emplois dans le secteur de l'assistance vers sa base et ses affiliés, en particulier les ex-prisonniers. Si cette activité ne représente pas une source d'accumulation, mais plutôt de redistribution des ressources, elle permet à la camorra de consolider sa légitimité sociale. Isaia Sales met en évidence la manière dont "les règles matérielles mêmes présidant à la distribution de ces flux d'assistance se prêtent à un contrôle camorriste. L'assistance comme forme de complément du revenu familial et comme distribution de richesse au-delà de la possession des conditions requises, ainsi que le manque de contrôle constituent des conditions facilitant l'entrée de la camorra dans ce domaine"(35).

Comme nous l'avons déjà constaté, la participation massive des entreprises camorristes aux marchés publics assurent de plus en plus l'accumulation de l'organisation camorriste, remplaçant progressivement le contrôle du trafic de stupéfiants. Le 
contrôle des flux financiers publics devient donc déterminant et la distribution politico-clientéliste des ressources, typique du système politique méridional, permettra qu'une substitution aisée - les équilibres entre les deux forces changeant - ne se fasse entre des hommes politiques et les camorristes en tant que redistributeurs des revenus de l'assistance étatique. Le rapprochement entre le milieu politico-administratif et la camorra, défini par G. Assante comme "le plus haut moment de l'imbrication entre pouvoirs criminels et pouvoirs institutionnels"(36), produit de véritables osmoses de personnes et de structures. D'une part, les nouvelles élites politiques - issues de moins en moins de la bourgeoisie des professions libérales mais plutôt de la nouvelle bourgeoisie des petits entrepreneurs, commerçants et professionnels de la politique sont souvent représentées par des individus sans scrupules qui adoptent des méthodes camorristes(37). D'autre part, la nécessité pour la camorra de s'approprier des opportunités générées par les flux d'argent public (outre celle de se garantir des marges d'impunité pour ses activités illégales) passe forcément par la recherche d'alliances à l'intérieur des centres publics de décision publique. D'ailleurs, si au départ l'alternance alliances/conf1its d'intérêts concerne la gestion irrégulière ou illégale des ressources publiques, par la suite, avec l'entrée de la camorra dans les circuits économiques légaux, des rapports de collaboration s'instaurent aussi dans des activités légales. En 1989, des enquêtes sur les administrations communales de plusieurs petites villes de la province napolitaine ont permis de dévoiler les liens existant entre certains élus et des représentants de la camorra.

Dans l'une de ces villes, Casandrino, des hommes politiques et administrateurs publics ont été dénoncés pour participation à la camorra. I1 y eut même une réunion, imposée par le "boss" camorriste aux représentants locaux des partis et des administrations publiques, afin de déterminer la composition du Conseil municipal. Domenico Di Maro, prête-nom de Nuvoletta, chef de l'une des plus importantes familles de la Nouvelle Famille, au moment de son arrestation, était conseiller municipal à Marano, zone contrôlée par la famille Nuvoletta elle-même. La Commission parlementaire d'enquête parle, en effet, de l'"insertion de la camorra dans le processus de formation des majorités politiques dans certains conseils municipaux avec le but évident d'orienter l'adjudication des marchés publics dans le domaine du bâtiment" et "d'insertion de la camorra dans certains conseils municipaux"(38). La présence d'élus camorristes dans les municipalités de l'arrière-pays napolitain paraît donc être tolérée par l'appareil politique local. Ce phénomène est d'ailleurs en train de se structurer, en devenant de plus en plus marqué lors de chaque élection.

Le décret Taurianova

Si la participation directe de la camorra à la vie politique locale est un fait, il est malgré tout trop tôt pour en évaluer la portée. Il est effectivement difficile, dans une réalité complexe comme celle de la région napolitaine, de cerner les frontières entre économie et politique, et de comprendre s'il s'agit d'un processus de légitimation politique croissante sur le mode de la mafia, ou seulement d'une modalité instrumentale pour le contrôle de l'économie publique, la camorra n'ayant pas réussi, en raison de la réaction des institutions politiques et administratives, à se constituer en "lobby économicopolitico-criminel" comme l'affirme A. Lamberti ?(39) On constate toutefois qu'il parait y avoir, de la part de la classe politique italienne, une réaction, que les implications politiques de la mafia, bien plus anciennes et importantes, n'ont jamais réussi à engendrer. Le 31 mai 1991, sur proposition du ministre de l'Intérieur, le gouvernement italien a promulgué un décret, confirmé ensuite par une loi(40), permettant au 
président de la République, sur proposition du ministre de l'Intérieur et avec délibération du Conseil des ministres, de dissoudre par décret les administrations locales dont les élus ont des liens directs ou indirects avec les organisations de type mafieux, ou dont le fonctionnement est compromis par les pressions exercées par ces dernières sur les administrateurs. Une commission extraordinaire est alors nommée pour la gestion municipale jusqu'aux nouvelles élections. La procédure est initiée par le préfet, gui peut demander au Parquet des informations constituées lors des dossiers judiciaires, et ce par dérogation au principe du secret, imposé par l'article 329 du code de procédure pénale.

Il semblerait que cette mesure ait été prise à la suite des remous provoqués par la qualification d'appartenance à la 'ndrangheta certains membres du Conseil municipal de Taurianova, en Calabre, dont la Commission parlementaire d'enquête avait demandé la dissolution. Or, il est vrai que le niveau de violence développé récemment par la 'ndrangheta calabraise est devenu inquiétant. Cependant, ces dernières années, les cas de compromis entre milieux politiques et milieux criminels, voire la participation politique directe de ces derniers aux instances politiques les, ont particulièrement concerné la camorra.

En ce qui concerne la mafia, sa participation aux instances polies locales remonte à plusieurs décennies et ses implications au niveau des institutions centrales de l'État ont été mises en évidence, à plusieurs reprises et depuis des années, par des journalistes et des politologues. Plusieurs repentis ont signalé des noms d'hommes politiques et de ministres : toutefois, aucune enquête judiciaire n'a pu, jusqu'à présent, percer l'écran des "raisons d'État" qui protège les hauts milieux politiques italiens.

Doit-on en déduire que l'attitude gouvernementale est à deux vitesses - des années d'acquiescement face à la mafia, et une réaction rapide vis-à-vis de la `ndrangheta et de la camorra? Cela pourrait refléter en effet une relation différente, ou une distance différente, des diverses organisations avec l'appareil central du pouvoir étatique : la raison de cette différence relationnelle pouvant être aisément trouvée dans l'accès plus récent à la participation politique de la part des deux dernières organisations.

Quelles peuvent être, cependant, les raisons dune réaction institutionnelle qui n'intervient que lorsque 'ndrangheta et camorra posent problème? Ne pourrait-il y avoir eu des pressions de la part des lobbies politico-mafieux, prêts à partager marchés criminels et marchés publics locaux, mais non une légitimité politique qui en s'inscrivant dans la durée, par remontée de la périphérie vers le centre, obligerait à partager le contrôle des équilibres et des règles mêmes du partage économique ? Une réponse partielle proviendra des critères d'application du décret: pour l'instant, ont été dissoutes les administrations municipales de Casandrino et de Taurianova.

Ce décret pose en tout cas de sérieux problèmes de fonctionnement démocratique. En effet, ou bien une procédure judiciaire aura prouvé l'appartenance ou les liens d'un élu ou d'un fonctionnaire avec les organisations de type mafieux - et donc le décret sera inutile -, ou bien on n'aura aucune preuve de cette appartenance ou de ces liens - donc le décret s'appliquera sur la base de simples rumeurs. Comment éviter, alors, qu'il ne soit utilisé pour régler des comptes politiques ou réaffirmer le contrôle de la hiérarchie d'un parti, au lieu de servir à éloigner les membres d'organisations de type mafieux des institutions politiques ? Ce décret s'insère, par ailleurs, dans une pratique qui a déplacé, de la "lutte contre le terrorisme" à la "lutte contre les organisations criminelles", 
l'utilisation d'instruments juridiques d'exception aux règles fondamentales du droit pénal et au respect des droits des citoyens et aux principes constitutionnels.

Conclusions

92 Le problème des infiltrations mafieuses dans le pouvoir central nous ramène donc aux relations entre organisations de type mafieux et État.

En ce qui concerne l'état du débat italien, le problème d'un "excès" ou d'un "vide" étatique en tant que facteur de développement du phénomène camorriste nous paraît représenter une approche biaisée, tout comme celles des organisations de type mafieux comme pouvoir parallèle, État dans l'État, ou anti-État. En fait, dans le Mezzogiorno, l'État a été en même temps excessivement présent - d'abord par l'hypertrophie administrative et la structuration d'une économie fondée sur les ressources publiques, ensuite par une focalisation répressive - et excessivement absent par le non développement de politiques sociales adéquates et de politiques saines de soutien à l'appareil productif. Présent et absent, l'État a été inefficace à tous les niveaux. Plus généralement, l'institution étatique s'est vidée de son sens au cours des quinze dernières années. Il existe désormais une coupure entre État formel et État réel, s'ils ont jamais coïncidé. La présence et la représentation dans le second de nombre d'intérêts divers et privés a sûrement été un facteur propice à la constitution de la camorra elle-même en tant que pouvoir réel.

En ce qui concerne la mafia, qui, la première, a tissé des relations avec le pouvoir central, il nous semble impossible d'établir une règle selon laquelle c'est elle qui s'est infiltrée dans les institutions ou ces dernières qui l'ont utilisée et renforcée. Il s'agit plutôt à notre avis, d'une fonction d'utilité réciproque, qui produit des mélanges et des recettes différentes, où parfois des intérêts convergents ont produit une correspondance des deux rôles pour les mêmes individus. A qui affirme que les institutions sont gangrenées par les organisations de type mafieux, on peut répondre que certains auteurs émettent l'hypothèse que c'est le développement au niveau politique, de la relation de "clientèle qui a créé et crée les potentialités sociales, économiques, politiques et institutionnelles à l'intérieur desquelles le comportement mafieux a trouvé et trouve aliment et modalités de reproduction, transformation et stratification"(41). Cependant, dans l'ensemble du Mezzogiorno, le vrai problème se situe à notre avis ailleurs : bien avant cette évolution du panorama politique italien, aucun des intérêts qui ont produit les politiques publiques pour le Mezzogiorno et aucun de ceux qui les ont appliquées n'avaient comme but le développement global du sud de l'Italie. Car, c'est d'abord la possibilité d'asseoir son pouvoir sur le tissu social et économique, assurée à la camorra par la précarité (ou la précarisation) des conditions socio-économiques de la région, qui lui a permis de structurer son organisation et de prétendre toucher directement aux enjeux politiques.

Mais nous ne voudrions pas prêter à équivoque. Si la camorra a pu devenir une organisation d'envergure et s'enraciner économiquement grâce à la dégradation du cadre socio-économique méridional, elle n'est pas, selon nous, "fille de la pauvreté et de la crise économique", mais organisme tout à fait fonctionnel au regard de la structure des pouvoirs publics et des intérêts des différents centres de pouvoir, ainsi qu'ils se présentent en Italie au niveau central et décentralisé. Il s'agit donc bien d'un "sous-développement administré", selon la définition de Nando Dalla Chiesa(42), car le développement d'un phénomène comme celui de la camorra est fonctionnel par rapport à une conception de la politique comme facteur général de régulation, qui 
s'assoit sur un système clientéliste fondé sur le sous-développement ; ainsi, le facteur de régulation supplémentaire "camorra" permet qu'aucune demande, association, organisation ou révolte au sein de la société ne mette en péril cet équilibre en exigeant un modèle de développement global, équilibré et équitable, ce qui entraînerait la fin du système lui-même. C'est bien l'État italien dans son actuelle matérialité, dans son mode de fonctionnement, dans ses représentations locales, ainsi que le système politique dans son ensemble, qui doivent donc être l'objet d'analyses et d'interventions(43). D'ailleurs, encore une fois avec le "décret Taurianova", l'intervention étatique s'attaque aux épiphénomènes au lieu de s'attaquer aux causes structurelles d'un malaise politique et économique avant tout : il faut croire que, dans le décalage croissant entre institutions politiques et société, les institutions politiques ne peuvent asseoir leur légitimité que sur des apparences.

\section{RÉSUMÉS}

Après avoir rappelé que la mafia sicilienne n'est pas l'unique modèle d'organisation de type mafieux en Italie même, l'auteur centre son analyse sur la camorra napolitaine. A travers une analyse historique qui insiste sur la légitimation populaire de l'organisation et son rôle supplétif à l'État dans la gestion de la violence et du contrôle social, l'auteur montre comment le trafic de drogue puis l'argent issu du tremblement de terre de 1980 ont modifié les structures traditionnelles. La camorra subordonnée à la mafia a pu grâce à l'argent de la drogue s'autonomiser par rapport à celle-ci et au pouvoir, même si ce trafic de drogue l'a amené à perdre une partie de son caractère populaire et à se scinder en deux organisations différentes. Mais, depuis les années 80, la camorra s'est désengagée du trafic de drogue au profit d'un investissement dans la gestion des fonds des caisses locales et des fonds d'aide aux mains des autorités décentralisées. Agissant par pression sur les entreprises ou directement en liai]on avec certaines autorités locales, la camorra est passée d'une coïncidence d'intérêt avec ces autorités dans la gestion de l'illégalité jusqu'à une osmose Qui lui permet d'agir en toute légalité. Le décret Taurianova qui vise à destituer les autorités locales soupçonnées de lien avec des groupes mafieux peut être lu soit comme une réponse à ces problèmes, soit plutôt comme une action des groupes politiques liés à la mafia sicilienne pour contrecarrer la montée en puissance de la camorra. Qu'en est-il alors du rapport qu'entretient l'État italien au Mezzogiorno et des politiques antimafieuses à mener?

the Sicilian Mafia is not the only Mafia-type organisation model in Italy, the author centres her article on the Napolitan Camorra. Using a historical analysis that insists on the organisation's popular legitimacy and on its role as a substitute to government in the management of violence and in social control, the author shows how drug trafficking and money stemming from the 1980 earthquake modified traditional structures. Camorra, at first subordinated to Mafia, managed, through drug money, to conquer some independence from government and Mafia itself, even though this drug-trafficking caused a loss of its popular support and a separation into two different organisations. But, since the 1980s, Camorra has disengaged itself from the drug traffic investing itself in the management of local and help funds in the hands of the decentralised authorities. Acting forcefully on local companies or directly linked to local authorities, Camorra 
has evolved from a position of common interest with these authorities in the management of illegality to an osmosis that allows for it to act legally. The Taurianova decree whose aim is to dismiss local authorities suspected of having links with Mafia groups, can be interpreted as either an answer to these problems or, more likely, as an action of political groups linked to Sicilian Mafia to thwart Camorra's growing power. What, therefore, should be thought of the links between the Italian government and the Mezzogiorno and of the anti-Mafia policies to lead ?

INDEX

Index géographique : Italie, méditerranée

Mots-clés : drogue, mafias et crime organisé, contrebande et trafic, économie politique

\section{AUTEUR}

\section{MARIA-LUISA CESONI}

Avocate, Assitante au Centre d'Etudes et Techniques et d'Evaluation Législatives, Faculté de droit de Genève 